\title{
A SIMPLE MECHANICAL MEASUREMENT FOR PRESSURE DISTRIBUTIONS AND FLOW RATES OF THORACIC AORTA
}

\author{
YANG-YaO NiU, NANG-Ping Kan \\ Institute of Mechanical and Aerospace Engineering, Chung Hua University, \\ Hsin-Chu, Taiwan
}

\begin{abstract}
The aim of this research is to design a simple three-dimensional aortic model to measure the relation of aortic pressure distributions and blood flow rate at the outlets of the aorta. The current experiment considers an area-varying curved tube with one branch to mimic an aortic arch. The measurement utilizes a water pump to substitute for the heart pumping task and collects data for the distributions of the pressure and flow rate relied on a PC-controlled pressure sensor. Various pressures as 160,140,110 and $90 \mathrm{~mm} \mathrm{Hg}$ are chosen as aortic entrance blood pressure conditions for the test cases. The pressure distributions from the current measurement will be used for the numerical boundary condition which can not be obtained from the current image technology
\end{abstract}

Biomed Eng Appl Basis Comm, 2005(October); 17: 252-257.

\section{INTRODUCTION}

The circulatory system in a human body is primarily a closed duct system. The aorta is the most important artery in a systematic circulation. It is divided into three sections, namely the ascending aorta, the aortic arch, and the descending aorta. The descending aorta may be further divided into two portions; namely the thoracic aorta and the abdominal aorta. The ascending aorta starts from the left ventricle and extends to the aortic arch with the branches like the carotid artery and the right carotid artery. The thoracic aorta starts from the aortic arch to descend along the spine before it joins the abdominal aorta. With the circulatory system, nutrients are conveyed by the blood to every corner of the human body. Blood pressure control is essential to the prevention of

Received: March 20, 2005; Accepted: August 18, 2005 Correspondence: Yang-Yao Niu, Professor

Institute of Mechanical and Aerospace Engineering, Chung Hua University, Hsin-Chu, Taiwan

E-mail: yniu@chu.edu.tw arteriosclerosis. The focus of this paper is about how to create a model of an aortic arch with a branch to mimic an aortic model containing an ascending aorta and a descending aorta; also using a water pump to substitute for the heart in order to measure ideal and non-ideal flow rates and pressure distributions on the inlet the ascending aorta and the outlet of the descending aorta as well as in the carotid artery. During the past years, many efforts have been done on the researches of the aorta and the carotid arteries. Regarding the aortic arch, Kenkichi, Ohba, et al. [1] measured the outline of the speed of palpitation in a model of aortic arch using laser induced fluorescence (LIF). There was plenty of research studies on the effects of arterial branching angles on the blood flow phenomena. F. J. H. Gijsen, et al. [2] conducted an experiment to compare nonNewtonian fluid with Newtonian fluid, for their physical discrepancies, while both of them were flowing in a tube with a $90^{\circ}$ curvature. Similarly, Y. I. Cho, et al. [3] simulated the changes of Reynolds number and pressure in human arterial branches with various angles $\left(30^{\circ}, 60^{\circ}\right.$ and $\left.90^{\circ}\right)$ and explored the meaning of such changes; the research was intended to display the separated flow inside an artery near the juncture of a branch, so as to measure the pressure 
levels with different angles and Reynolds numbers, consequently revealed the important effects caused by arterial angles. Donald F. Foung, et al. [4] studied the effect of blood flow on the shape and appearance of the constriction of constricted vessels, with Reynolds numbers ranging between 100 and 5000. V. Deplano, et al. [5] analyzed the wall stress of a constriction model using Doppler ultrasonic velocimeter. M. Siouffi, et al. [6] conducted an experiment to understand the influence of an inlet flow rate on blood flowing through a passage characterized by a $75 \%$ degree of stenosis. Deshpande, et al. [7] calculated the circulation of a steady flow in a symmetrically stenosised vessel and discovered that the findings conformed mostly to the results of a similar experiment conducted by Young and Tsai [8]. H. Liu, et al. [9] studied the inlet flow rate on the characteristics of the driving force in a twodimensional stenosised channel.

\section{METHOD}

\subsection{Experiment Facility}

This experiment was designed to simulate the physical phenomena of the pressure and the blood flow in the ascending aorta, the descending aorta and the carotid arteries of a human body under normal blood pressure and abnormal blood pressure (hypertension) respectively. Hence, much consideration was given to the planning and preparatory work. Major equipment included sensors, a model of gradual stenosis of the aortic arch, wave source generating devices, retrieval and analysis devices, and a sink. Descriptions of the major devices used in this experiment are as follows:

\section{A. Sensors}

(a) Pressure in the ascending aorta (at the inlet), the descending aorta (at the outlet), and the carotid artery (in the branch) was measured, using two BD sensors (1 bar).

(b) Flow was measured with load cells. Variations of flow in the descending aorta (at the outlet) and the carotid artery (in the branch) were measured, using AND LCBO3K015M 26.00.

\section{B. Model of Gradual Stenosis of the Aortic Arch}

According to the literature, the diameter of the aorta is between 2.6 and $3.5 \mathrm{~cm}$, and the aorta tapers off. Hence, the aforesaid specifications were observed during the fabrication process, producing a model composed of an ascending aorta (the inlet), a descending aorta (the outlet) and a carotid artery (the branch) and made from brass. The inlet of the aorta is $30 \mathrm{~mm}$ in diameter, whereas the outlet of the aorta is $22 \mathrm{~mm}$ in diameter. The inlet of the arterial branch is
$20 \mathrm{~mm}$, whereas the outlet of the arterial branch is $18 \mathrm{~mm}$. The caliber model is characterized by tapered dimensions. Fig. 1 shows the completed aortic arch model.

\section{Wave Source Generating Devices}

Generation of a wave source is the key to success in this research. As regards a pulse, systolic duration is around 0.25 second, whereas diastolic duration is around 0.6 second. Hence, any simulation has to meet the aforesaid requirements. In this experiment, the devices for generating a wave source include the following.

(a) Immersion Water Pump

This experiment used a Walrus immersion water pump PW100 which has a caliber of one inch and a maximum head of $6.5 \mathrm{M}$. The system employed the water pump to simulate the kinetic energy the heart requires to convey blood.

(b) Power Adjuster control machine

This experiment adopted a Dai Yi Shing power adjuster, 50/60-Input, 0 140 Max 5A, to adjust the operating strength of the water pump and thereby adjust and set the pressure at the inlet of the aorta for the sake of measurement.

(c) Actuator

This experiment used a Taian Electric CN-11 actuator (take off if required), $110 \mathrm{~V}, 50 \sim 60 \mathrm{~Hz}$ to turn on/off the water pump and enable the water pump to produce a wave source.

(d) Intermittent Controller

This experiment used the RTDV AC/110/220 CONNECTION DIAGRAN TIME DELAY CONTACT 3A NON-INDUCTIVE LOAD 220VAC $50 / 60 \mathrm{~Hz}$ and made good use of its adjustable On/Off feature. This, coupled with the actuator, met the demand for controlling the On/Off operation of the water pump in the system.

(e) Sink

A sink, whose dimensions were $45 \mathrm{~cm}$ long, $45 \mathrm{~cm}$ wide, and $45 \mathrm{~cm}$ high, was independently made from steel. The inside of the sink was divided into two compartments to allow for the circulation of fluids and

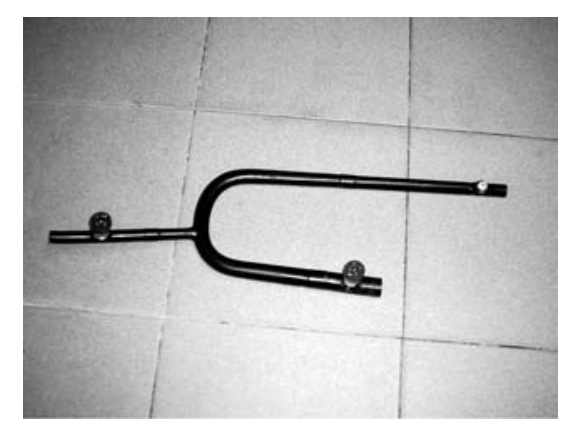

Fig 1. The diagram of an aortic arch model. 
function as basic devices for positioning and measuring the tapering aortic arch model, the water pump, and the load cells.

\subsection{Procedure of Measurement}

This system used the water pump to simulate the motion of the heart pumping. This system also used the power adjuster, the intermittent controller and the actuator so as to cause the water pump to generate pulse signals and retrieve/simulate the pressure and flow inside blood vessels through a pressure switch and the load cells. The retrieved data underwent conversion by means of an amplifier and an $\mathrm{AD} / \mathrm{DA}$ interface card before being read by a PC. As a result of the aforesaid process, this experiment simulated the physical characteristics of the pressure and the flow of the blood sent to the aorta and the branch of carotid artery by cardiac contraction. The intermittent controller, coupled with the actuator, generated the pulses. The intermittent controller regulated the operation and the cessation timing of the water pump in the light of the experimental requirements. The actuator controlled the operation and the cessation of the water pump through the triggering function of the intermittent controller. In this experiment, the movement of the intermittent controller was regulated in such a way that it moved for one second and then stopped for one second in order to control the water pump, generating pulse signals, with a view to simulating the relationship between the blood pressure and the blood flow of the aorta and the branch of carotid artery in a human body, as shown in Figure 2.

\subsection{Methodology of Experiment}

The primary purpose of this system was to measure three kinds of parameters, namely the pressure at the ascending aorta, the pressure and the flow at the descending aorta, and the pressure and the flow in the carotid artery. The method for measuring the aforesaid parameters is described as follows:

A. Measuring the Pressure at the Inlet of the Aorta

As regards measuring the pressure at the inlet of the aorta, it involved putting the system in the form of a full tube device first, and then connecting the respective outlets of the branch of carotid artery and the aorta to the sink with transparent plastic tubes, and then filling the whole testing system with water by siphon principle and through the operation of a water pump, and eventually tuning the pressure to the required level by adjusting the water pump with a power adjuster. This paper involves measuring pressure of $160 \mathrm{mmHg}, 140 \mathrm{mmHg}, 110 \mathrm{mmHg}$, and $90 \mathrm{mmHg}$. On acquisition of the correct pressure values intended for measurement, cardiac pulsation was simulated by controlling the water pump with the

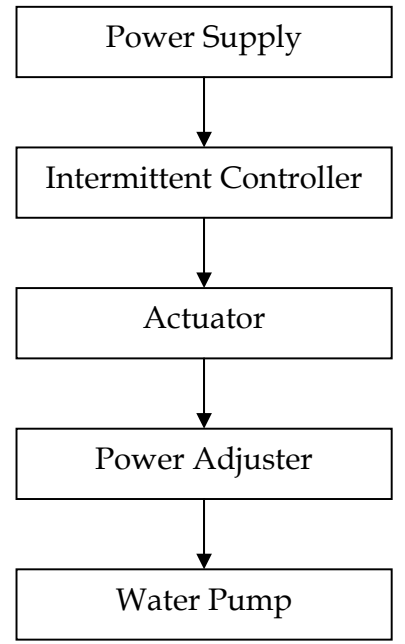

Fig 2. Charts for generation of pulse wave and method for controlling pressure

intermittent controller. In the course of measurement, operation lasted for one second and then paused for one second, thus pulse signals intended for measurement were generated as a result of the repeated on-off movements of the water pump. At this point, the pressure switch installed in the ascending aorta model began to read data, whereas self-compiled QB software began to read and store data for the sake of analysis of data.

B. Measuring the Pressure at the Outlet of the Aorta

The pressure at the outlet of the aorta was measured by estimating the pressure at the inlet of the ascending aorta. In this system, a pressure switch was also installed at the outlet of the aorta; to complete the measurement of the pressure at the inlet of the aorta, the pressure at the outlet of the aorta was measured by means of the identical inlet pressure level. Artery

C. Measuring the Pressure at a Branch of Carotid

A branch of carotid artery was installed above the aortic arch. It was equipped with a pressure switch. To perform the measurement of pressure at the outlet of the aorta, the branch of carotid artery was carried out with the aforementioned method, using the identical inlet pressure level. Figure 3 shows the positioning of pressure switches as

D. Measuring the Flow of the Aorta

Aortic flow was measured, using a load cell. Installed inside the sink of the system was a load cell. Above the load cell was a small sink intended to hold water. To prevent the weight of the water pump from affecting the measurement performed by the load cell, the water pump was hanged above the sink with a rope. To measure aortic flow, reference was made to the 


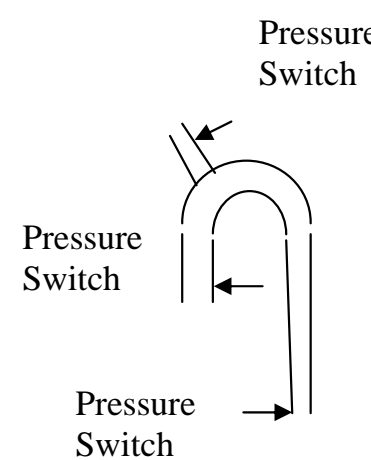

Fig 3 . A diagram of the design of positioning pressure switches

pressure taken at the inlet of the aorta, and then, on completion of the measurement of the intended pressure, the weight of the water driven to the aorta by the water pump was measured, using the same pressure conditions (as shown in Fig. 4). Any signal detected by the load cell was amplified by 200 times with the independently installed amplifier, and then the amplified signal which had been an analog signal heretofore was converted into a digital, logical signal to be read by computer, using an AD/DA interface card. Then, the digital, logical signal was read and recorded with self-compiled QB software for the sake of analysis of data.

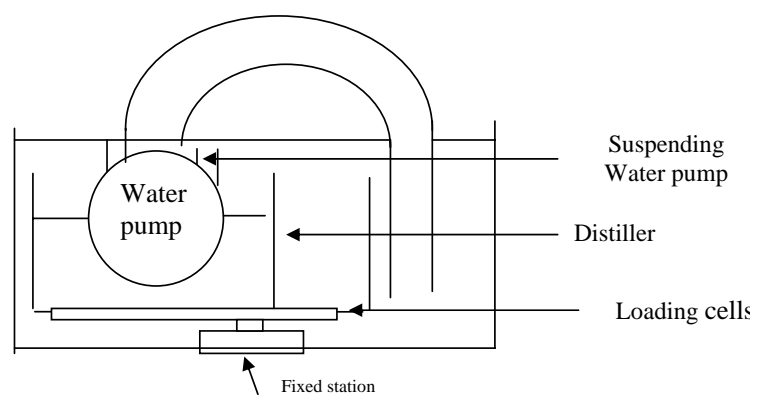

Fig 4. Measuring the flow in aorta design of positioning E.

E. Measuring the Flow in a Branch of Carotid

With the same pressure conditions, the flow in a branch of carotid artery was measured by making reference to the aortic inlet pressure. The method for measuring the flow in a branch of carotid artery was different from that for measuring the flow in the aorta; the latter involves using a load cell to calculate the weight of fluids driven by a water pump, whereas the former involves calculating the increase in the weight of fluids flowing into a sink. The former also involves connecting the outlet of the branch of carotid artery to a plastic tube, and then putting the plastic tube in the sink installed above the load cell. The method for controlling the water pump was identical with the counterpart used in measuring aortic flow. Once a pulse of the water pump produced water flow, the fluid would flow into the branch of carotid artery; then, the plastic tube connected to the branch of carotid artery would bring the water to the sink, and the water weight increment would represent the flow accumulated in the carotid artery. In the course of accumulation, data were read and recorded with software for the sake of analysis.

\section{RESULT}

To study the blood pressure in a normal aorta as well as the blood pressure in an abnormal aorta, this experiment made reference to the aortic inlet pressure. Hence, in this experiment, the first parameter to be measured was the aortic inlet pressure. After taking the correct values of the aortic inlet pressure, the researchers proceeded to measure the aortic outlet pressure and the pressure in the branch of carotid artery. This experiment was designed to study blood pressure in four different conditions, and the findings are as follows: The represented curves of the $160 \mathrm{mmHg}$ as aortic inlet pressure, the aortic outlet pressure, and the branch pressure are shown in Fig. 5-1 and $5-2$. The respective curves of the $140 \mathrm{mmHg}$ as aortic inlet pressure, the aortic outlet pressure, and the branch pressure are shown in Fig. 5-3 and 5-4. The respective curves of the $110 \mathrm{mmHg}$ as aortic inlet pressure, the aortic outlet pressure, and the branch pressure are shown in Fig. 5-5 and 5-6. The respective curves of the $90 \mathrm{mmHg}$ aortic inlet pressure, the aortic outlet pressure, and the branch pressure are shown in Fig. 5-7 and 5-8. The respective curves of the flow rate under various pressure conditions are shown in Fig. 59. From the measured data, the simultaneous recordings of pressure distributions at the entrance and the outlets of aorta and its branch are shown to be in a similar tendency with the physiological condition. It is always shown that the heart contracts, pressure rises rapidly at the beginning of the systole, then reaches the maximum peak of the pressure profile at the end of the systole and gradually decreases during the diastole. In Figure 5-9, we can find that the flow rate at the outlet of the aorta always reach the maximum peak around one second no matter what pressure at the inlet is chosen.

\section{CONCLUSION}

The current work is design a simple threedimensional aortic model to measure the relation of 


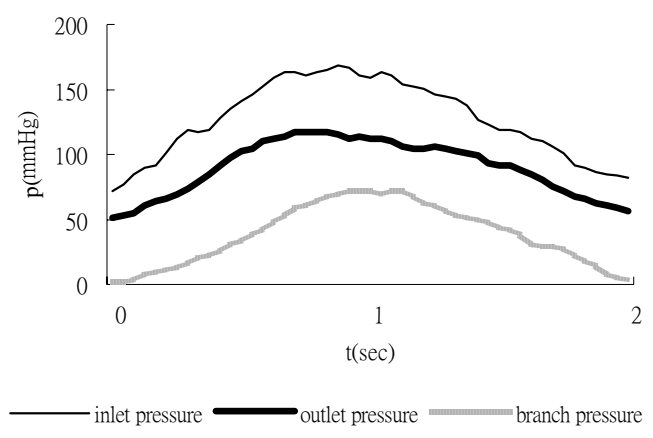

Fig 5-1. Distributions of aortic inlet pressure, outlet pressure, and the branch pressure for the $160 \mathrm{mmHg}$

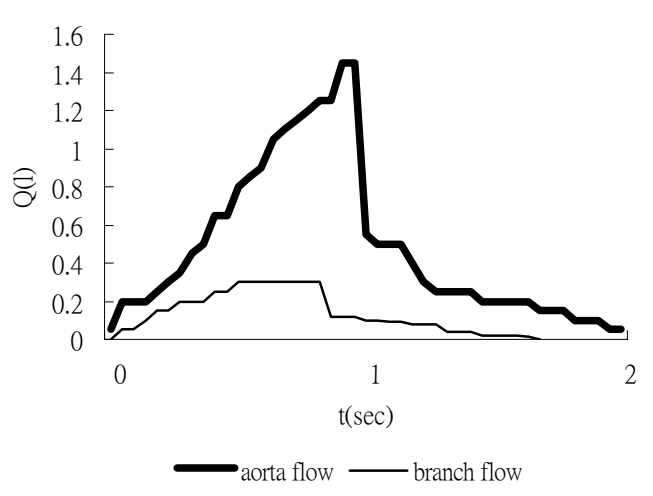

Fig 5-2. Flow rate distributions along the aorta and branch for $160 \mathrm{mmHg}$

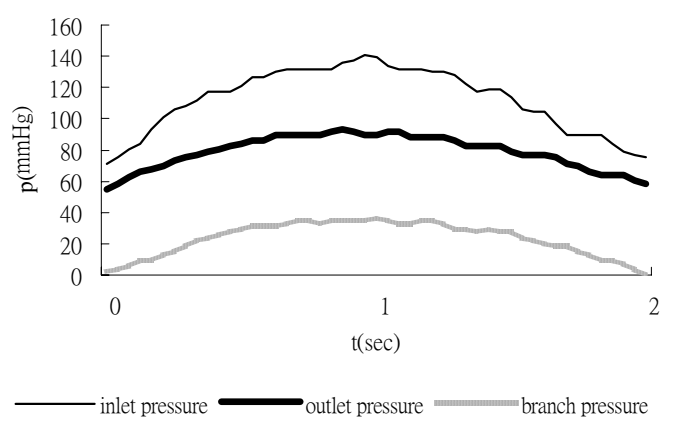

Fig 5-3. Distributions of aortic inlet pressure, outlet pressure, and the branch pressure for $160 \mathrm{mmHg}$

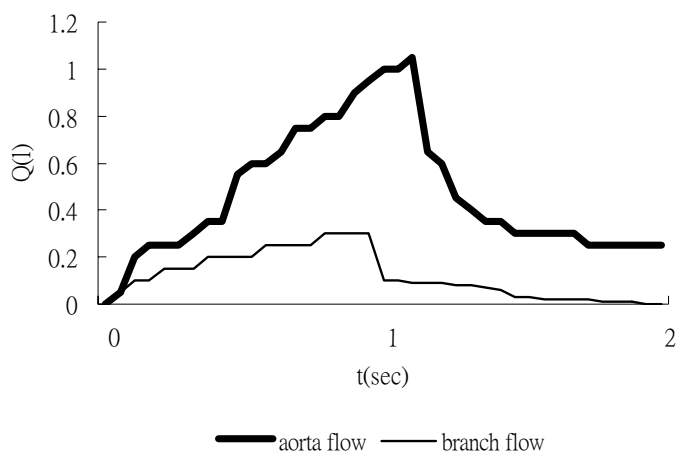

Fig 5-4. Flow rate distributions of aorta and branch for $140 \mathrm{mmHg}$

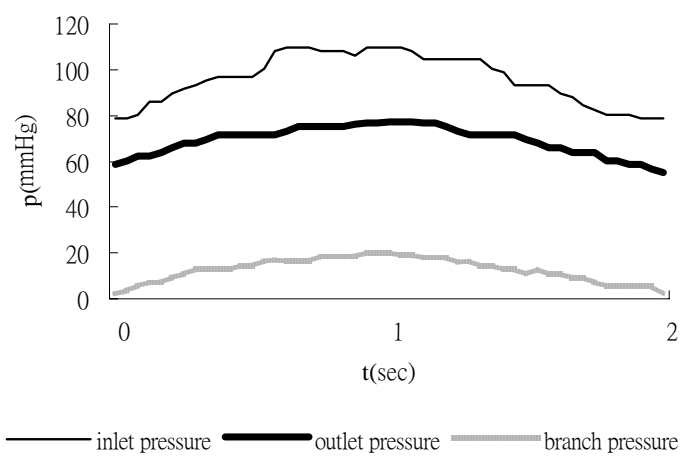

Fig 5-5. Respective curves of aortic inlet pressure, the aortic outlet pressure, and the branch pressure for $140 \mathrm{mmHg}$

aortic pressure distributions and blood flow rate at the outlets of the aorta. The current experiment considers an tapered and curved tube with one branch to mimic an aortic arch. From the measured data, the simultaneous recordings of pressure distributions at the entrance and the outlets of aorta and its branch are shown to be in a similar tendency with the physiological condition. It is shown that pressure always rises rapidly at the beginning of the systole, and then reaches the maximum peak of the pressure profile at the end of the systole and gradually decreases during the diastole. Also, we can find that the flow rate at the outlet of the aorta always reach the maximum peak around one second no matter what pressure at the inlet is chosen. The pressure distributions from the current measurement will be used for the numerical boundary condition which can not be obtained from the current image technology. 


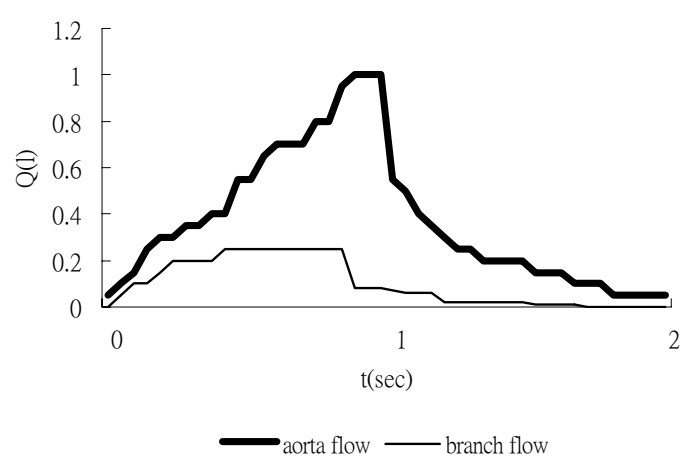

Fig 5-6. Flow rate distributions of aorta and branch for $110 \mathrm{mmHg}$

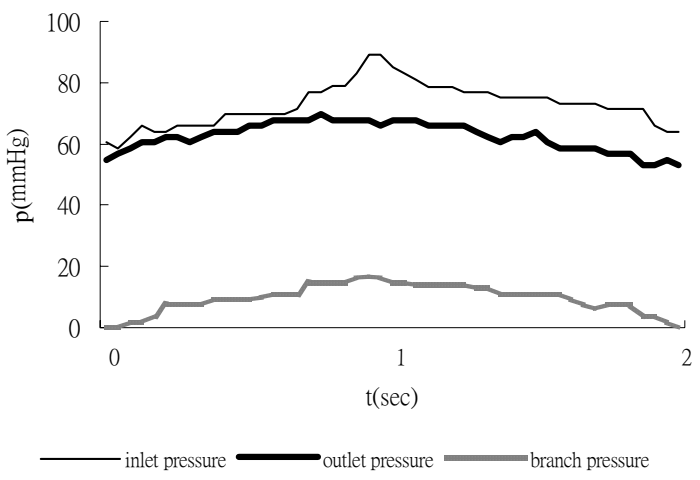

Fig 5-7. Respective curves of aortic inlet pressure, the aortic outlet pressure, and the branch pressure

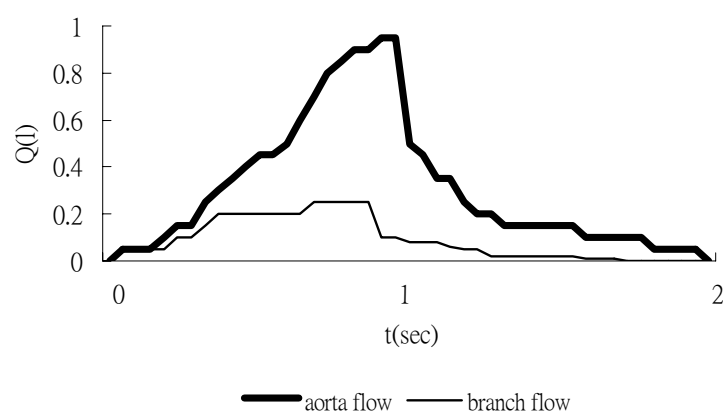

Fig 5-8. The flow rate distributions of aorta and branch for $110 \mathrm{mmHg}$

\section{ACKNOWLEDGEMENT}

The authors wish to acknowledge the financial support from the National Science Council under NSC91-2212-E-216-017.

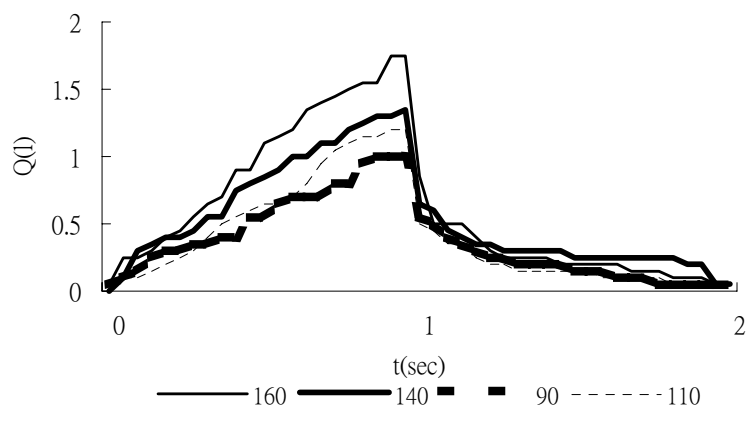

Fig 5-9. A comparison of the flow rate at the entrsance under various pressure conditions

\section{REFERENCE}

1. Ohba K, Bando K, Kamino H, Urabe T, lkedo S, and Fujita Y: Numerical Simulation and Experiment of Pulsaitle Flow in Modeled Aortic Arch, Pulasatile Flow in Modeled Aortic Arch, 119135.

2. Gijsen F J, Allanic E,Van de vosse F N ,Janssen J D : The influence of the non-Newtonian properties of blood on the flow in large arteries : unsteady flow in a $90^{\circ}$ curved tube, Journal of Biomechanics, 32:705-713, 1999.

3. Cho Y I, Back L H, Crawford D W: Experimental Investigation of Branch Flow Ratio, Angle, and Reynolds Number Effects on the Pressure and Flow Fields in Arterial Branch Models, Journal of Biomechanical Engineering, 107: 257-266, 1985.

4. Young D F , Tsai F Y: Flow Charactreristics in Models of arterial stenoses-1. steady flow J. of Biomechanics, 6:395-410, 1973.

5. Deplano V, Siouffi M: Experimental and numerical study of pulsatile flows through stenosis : Wall shear stress analysis, Journal of Biomechanics 32:1081-1090,1999.

6. Siouffi M, Deplano V, Pelissier R: Experimental analysis of unsteady flows through a stenosis, Journal of Biomechanics 31:11-19, 1998.

7. Dshpande M D, Giddens D P, Mabon F R: Steady laminar flow through modeled vascular stenoses. Journal of Biomechanics 9, 165-174, 1976.

8. Young D F, Tsai F Y: Flow characteristics in models of arterial stenosis I. Steady flow, Journal of Biomechanics 6:395-410, 1973.

9. Liu H, Yamaguchi T: Waveform dependence of pulsatile flow in a stenosed channel, Transaction of the ASME, J. of Biomechanical Eng, 123:88, 2001. 